\title{
Trivium
}

Revue franco-allemande de sciences humaines et sociales - Deutsch-französische Zeitschrift für Geistesund Sozialwissenschaften

$7 \mid 2010$

Max Weber et la bureaucratie

\section{Hat die Bürokratie eine Zukunft?}

\section{Patrice Duran}

Traducteur : Andreas Pfeuffer

\section{(2) OpenEdition}

\section{Journals}

\section{Édition électronique}

URL : http://journals.openedition.org/trivium/3819

DOI : $10.4000 /$ trivium.3819

ISSN : 1963-1820

\section{Éditeur}

Les éditions de la Maison des sciences de l'Homme

\section{Référence électronique}

Patrice Duran, « Hat die Bürokratie eine Zukunft? », Trivium [Online], 7 | 2010, online erschienen am 06 Dezember 2010, abgerufen am 08 September 2020. URL : http://journals.openedition.org/trivium/ 3819 ; DOl : https://doi.org/10.4000/trivium.3819

Ce document a été généré automatiquement le 8 septembre 2020

\section{(c) (i) () $\Theta$}

Les contenus des la revue Trivium sont mis à disposition selon les termes de la Licence Creative Commons Attribution - Pas d'Utilisation Commerciale - Pas de Modification 4.0 International. 


\section{Hat die Bürokratie eine Zukunft?}

\section{Patrice Duran}

Traduction : Andreas Pfeuffer

\section{NOTE DE L'ÉDITEUR}

Wir danken Patrice Duran für die freundliche Genehmigung, diesen Text für die vorliegende Ausgabe von Trivium zu übersetzen.

1 Nisbet legte völlig zu Recht Wert darauf, dass Webers Untersuchung der Rolle der Bürokratie »nicht einfach den Anknüpfungspunkt für aktuelle Untersuchungen darstellt. Bis auf wenige Ausnahmen fasst sie sie alle zusammen. Noch niemand hat bisher etwas formuliert, was nicht in der Weberschen Theorie oder vielmehr im Weberschen Herrschaftskonzept zumindest implizit schon angelegt ist. $\ll^{1}$ Diese Behauptung bleibt freilich insofern paradox, als ein Großteil der Untersuchungen zur Bürokratie ganz offen als Kritik der Weberschen Formulierung des Idealtypus der Bürokratie aufgetreten ist. Die historische, aber auch geistesgeschichtliche Bedeutung des Phänomens Bürokratie macht das eigenartige Unverständnis, ja die Fehleinschätzungen um so überraschender, denen die Arbeiten Max Webers zu diesem Thema außerhalb Deutschlands ausgesetzt gewesen sind. Es ist bezeichnend, wenn das Kapitel, das François Chazel der Weberschen Bürokratieauffassung 1995 widmet, bis heute den einzigen wirklich gut informierten französischsprachigen Text darstellt, der das Denken Webers in seiner Komplexität präzise und nuanciert nachvollzieht. ${ }^{2}$ Eine Reihe falscher Debatten ganz besonders innerhalb der Organisationssoziologie geht mithin von einer zumeist unvollständigen und einseitigen Interpretation des Werks dieses bedeutenden Soziologen aus.

Das Thema Bürokratie ist so sehr mit dem Werk Max Webers verbunden, dass die ganze organisations- oder verwaltungswissenschaftliche Literatur am Ende in ihm - fast schon bis zur Karikatur - ausschließlich den Anwalt einer einzigen Sache sehen wollte. Weber rein auf die Bürokratie zu reduzieren ist nicht nur ungerecht und Beleg für eine ungeheure Ignoranz, sondern schlicht absurd. Die Rationalisierung lässt sich nicht 
einfach auf die Bürokratie beschränken, es sei denn, man stellt Weber auf eine Ebene mit Taylor, was nicht wenige Managementspezialisten immer noch tun, die zu allem Unglück dafür noch Bestätigung bei March und Simon finden. ${ }^{3}$ Wenn man nur etwas genauer hinsieht, zeigt sich sehr wohl, dass man einen Autor, der ein so feines Gespür für soziales Handeln besaß und der auch auf dessen Komplexität und angesichts der potenziellen Fülle menschlicher Entscheidungsmöglichkeiten auf die nur geringe Vorhersehbarkeit menschlichen Handelns hinwies wie Weber, nicht mit einem Autor gleichsetzen kann, der dagegen ein Modell des Menschen erstellt, das ebenso armselig ist wie das des nutzenmaximierenden homo oeconomicus. Die Bürokratietheorie Webers entfaltet ihre ganze Bedeutung erst im Rahmen einer allgemeinen Handlungstheorie. Ausgehend von einer allgemeinen Soziologie hatte Weber eine Handlungstheorie entwickelt, mit der er organisiertes Handeln als einen zweckrationalen Handlungstypus entwerfen konnte. Darauf sollte man durchaus nochmals hinweisen. Wie François Chazel feststellt, ist die Webersche Sicht auf die Bürokratie »jedoch nicht ausreichend in sich geschlossen, als dass man sie in einer einzigen Theorie zusammenfassen könnte, sie lässt sich jedoch in einzelne, für sich stehende, freilich komplementäre Bestandteile zerlegen. $\aleph^{4}$ Einfach ausgedrückt, könnte man sagen, dass sich das, was Weber schreibt, auf zwei der Bürokratie eigene Dimensionen richtet, und zwar in dem Sinne, als diese zugleich Herrschaft, Herstellung einer legitimen sozialen Ordnung, und Macht, ein Modus der Arbeitsorganisation im Hinblick auf die Lösung praktischer Probleme, ist. Sie antwortet auf zwei Dinge, nämlich auf die Frage der Legitimität der sozialen Ordnung und auf die der Effizienz des Handelns. Weber zeigt die Dualität bürokratischer Organisation auf, womit er die Notwendigkeit betont, zwischen der Legitimität und der Effizienz als zwei analytisch $\mathrm{zu}$ trennenden Fragen $\mathrm{zu}$ unterscheiden. Die Dauerhaftigkeit formaler Organisationen ist eine Erklärung dafür, warum die Frage der Bürokratie in der Tat weiterhin die Organisationssoziologie in einem solchen Maße beschäftigt, dass es uns nötig erscheint, heute zumindest gerafft auf den Beitrag Max Webers einzugehen, wenn wir den offensichtlichen Widerspruch zwischen der Aufrechterhaltung bürokratischer Strukturen der Handlungskoordination und dem stetig wiederkehrenden Bemühen um deren Überwindung besser begreifen wollen.

Ohne uns allzu lang darüber auszulassen, müssen wir doch in groben Zügen auf die zentralen Punkte eingehen, die in unseren Augen den Kern des Denkens des deutschen Soziologen ausmachen. Er versteht es in seinen Arbeiten, den engen Zusammenhang zwischen den Glaubensvorstellungen, den Organisationsformen und den Wirtschaftssystemen aufzuzeigen und dabei noch soziologische und historische Kausalität auseinanderzuhalten. Vergessen wir nicht, dass Weber das Aufzeigen von Regelmäßigkeiten im sozialen Leben und die Erklärung von historischen Einzelereignissen verbinden will. Er beschränkt sich nicht auf eine schlicht chronologische Perspektive, sondern betont die wechselseitige Abhängigkeit der gesellschaftlichen Erscheinungen. Der Webersche Ansatz verfährt genetisch und zugleich funktional. Wenn sich die Bürokratie als die Institutionalisierung zweckrationalen Handelns fassen lässt, dann sollte man ihr Werden in ihrer historischen Dimension begreifen, und das heißt, wie François Chazel nachdrücklich betont, im Kontrast $\mathrm{zu}$ den patriarchalischen Strukturen und der patrimonialen Verwaltung. Die Bürokratie ist die Konkretisierung des sich entfaltenden Rationalisierungsprozesses, den Weber im Abendland am Werk sieht, denn er orientiert seine Untersuchung der Entwicklung des okzidentalen Kapitalismus ja an der Rationalität. ${ }^{5}$ Mühelos zeigt er die unbestreitbare Leistung der bürokratischen 
Organisation für die Entfaltung des Kapitalismus auf. Giddens betont das im Übrigen klar, wenn er schreibt, dass der rational organisierte Staat bei Max Weber nicht nur eine "Folge " des sich ausbildenden modernen Kapitalismus ist, sondern seiner Entstehung vorausgeht und seine Entwicklung begünstigt. ${ }^{6}$ Die Bürokratie spielt nämlich just dadurch eine bedeutende Rolle für die Entstehung des modernen Staates, dass sie die gesellschaftliche Demokratisierung und Professionalisierung seines Personals aufgrund des der Qualifikation eingeräumten besonderen Gewichts sicherstellt. Darin zeigt sich die Bedeutung der Untersuchungen, in denen Weber den Beweis dafür erbringt, dass die Disziplin die militärische Nutzung des Schießpulvers erklärt und nicht umgekehrt. Der sachgemäße Einsatz der Mittel, insbesondere der menschlichen, erzwingt die Aufgabe jeglicher Form von Patrimonialismus. Doch wenn sich die Bürokratie als eine spezifische institutionelle Form analysieren lässt, deren Genese und Eigentümlichkeiten man verstehen muss, dann muss sie gleichermaßen als eine Form der Arbeitsteilung gesehen werden, deren Leistungsfähigkeit wie auch Grenzen zu analysieren sind. Eine oberflächliche Lesart des Weberschen Idealtypus mag zwar nahelegen, es gebe nur eine einzige bürokratische Form, besinnt man sich hingegen darauf, dass der Bürokratiebegriff ein historisch gewonnenes Konzept und kein Oberbegriff ist, dann lässt sich darin auch der Ausdruck einer Relativierung der Bürokratie in zweifacher Hinsicht sehen: zum einen hinsichtlich der Einheitlichkeit ihrer Formen, zum anderen der Qualität ihres Funktionierens. Man sollte sich nämlich davor hüten, eine einfache Opposition zwischen einem für die Verschiedenartigkeit der gesellschaftlichen Formen empfänglichen Weber und einem nur in vereinfachenden Idealtypen denkenden Weber aufzubauen. Wenn sich die Rationalität als eine der zentralen Dimensionen der abendländischen Kultur durchsetzt, bedeutet das nicht, dass es eine völlige Ähnlichkeit der empirisch $\mathrm{zu}$ konstatierenden Rationalisierungsprozesse gibt. Dass bürokratische Organisationsprinzipien sich tendenziell über den gesamten Westen ausbreiten, heißt keineswegs, dass ihre praktische Ausgestaltung und ihre Funktionsweise überall die gleichen sein müssten. Die von Weber postulierte Einheitlichkeit der westlichen Welt schließt zu keinem Augenblick interne Differenzen aus. Diese Einheitlichkeit macht nur in Bezug auf den Rest der Welt Sinn. Mag man auch bisweilen ein gewisses Quantum an Evolutionismus bedauern, so kann man doch nicht die mit Finesse durchgeführten Analysen unter den Tisch fallen lassen, in denen Weber die im Westen selbst auftretenden Unterschiede, insbesondere hinsichtlich der Realität und der Vielfalt der institutionellen Arrangements, $\mathrm{zu}$ fassen verstand. ${ }^{7}$

4 Ursache und Funktion sind auf jeden Fall zu unterscheiden, und das Auftauchen einer gesellschaftlichen Form erklärt nicht automatisch ihre Beibehaltung - darin stimmen Durkheim und Weber überein. Der Kapitalismus ist bekanntermaßen weniger der Profit an sich, sondern dessen überlegter Einsatz zu Investitionszwecken unter Zuhilfenahme ökonomischen Kalküls. Das Eigentümliche der rational-legalen Regeln und der dazugehörigen Legitimität besteht darin, dass sie ein Minimum an Stabilität gewährleisten und so Antizipation und Vorausschau möglich machen, dass man nötigenfalls aber auch die Konstellation der Bedingungen von Produktion und Tausch in dem Maße verändern kann, wie sich die Regeln selbst in Abhängigkeit von präzisen Regeln verändern lassen. Manche wollten darin das Vorliegen einer »strukturalen Homologie» sehen, der zufolge die der bürokratischen Organisation zugrunde liegenden Prinzipien in enger Entsprechung zum Funktionieren der kapitalistischen Wirtschaft stünden. ${ }^{8}$ Aufgrund der dem Begriff innewohnenden Schwierigkeiten neigen 
wir jedoch zur Vorsicht, und vielleicht böte sich hier die Verwendung des Weberschen Begriffs der "Adäquatheit" eher an, um den Zusammenhang zwischen den beiden Phänomenen begrifflich zu fassen. Ein Qualitätsmerkmal von Unternehmen ist es insbesondere, Stabilität und Wandel zusammenzudenken, da sich der Kapitalismus bei Weber wie bei Marx durch die unablässige Umwälzung der Produktionsverhältnisse und der Produktivkräfte auszeichnet. Der Kapitalismus darf nicht erstarren, und das erfordert Flexibilität seitens der Produktionsmittel wie der Menschen. Daher darf beispielsweise der kapitalistische Unternehmer nicht aufgrund irgendwelcher Anhänglichkeiten Gefangener seiner eigenen Angestellten sein. Da die Trennung des Arbeiters von den Betriebsmitteln ein Strukturmerkmal der Industriegesellschaften darstellt, kann eine Anstellung in einer Welt, in der man Kompetenz kaufen muss, nicht auf der Vorrangstellung sozialer Bande beruhen. Genau aus diesem Grund lässt sich das Aufkommen der Bürokratie in Webers Augen nicht von der Geldwirtschaft lösen, ebenso wenig aber auch vom Recht, das aus jedem Menschen einen »formal freien Arbeiter « macht. Diese Dimension ist grundlegend, und die Logik des Arbeitsvertrags kann bei Weber nicht einfach als ein ideologischer Fallstrick analysiert werden, dem es darum ginge, der kapitalistischen Herrschaft den schönen Schein der Gleichheit zu verleihen. Hier denken Weber und Marx vollkommen unterschiedlich; während dieser die sozialen Verhältnisse in ihrer Entwicklungsabfolge analysiert, erklärt jener die Institutionen, die für die Fortschritte der Rationalisierung sorgen, ohne dass er deswegen für die möglicherweise damit einhergehenden Herrschaftseffekte blind wäre. Die Bürokratie muss eine Lösung für das Problem der Kooperation und der Integration ganzer Gruppen von Akteuren bieten, die nun nicht mehr über soziale Attribute miteinander verbunden sind. Die Regeln werden $\mathrm{zu}$ wirkungsvollen Integrationsprinzipien, da das einzige, was von den Individuen verlangt wird, Kompetenz ist. Der freie Arbeitsmarkt ist eine der wesentlichen Voraussetzungen für das reibungslose Funktionieren der kapitalistischen Ökonomie und weitergehend der industriellen Welt. Das Schwächerwerden patriarchaler Bande wie auch der gemeinschaftlichen Dimensionen des Gesellschaftslebens wirft infolgedessen das Problem der Autonomie der Herrschaft gegenüber den sozialen Formen auf, wobei die Bürokratie diesem »disembedding" der Herrschaft aus den "ererbten Privilegien«, wie Nisbet sie treffend genannt hat, entspricht. ${ }^{9}$ Hier zeigt sich das Bemühen Webers um ein Verständnis dessen, wie aus sozialem Handeln hervorgehende Beziehungen soziale Ordnung generieren. Es geht ihm nämlich darum, die Herausbildung einer legitimen Ordnung zu erklären. Die Ausdehnung der bürokratischen Organisation beruht auf der Fähigkeit, sich in zweierlei Hinsicht freizumachen, nämlich von Face-to-faceBeziehungen wie von den gesellschaftlichen Verhältnissen. Im Grunde genommen geht es um die Frage, wie man sich vom Sozialen frei machen kann, um effizientes Handeln zu erreichen. Dieses Erfordernis zeigt sich übrigens in sämtlichen gesellschaftlichen Sphären - und darin offenbart sich denn auch einer der typischen Züge der Moderne -, was erklärt, warum die Bürokratie die Grenzen zwischen Öffentlich und Privat übersteigt.

5 Mittels Bürokratie lassen sich also gesellschaftliche Verhältnisse verwalten wie auch praktische Probleme lösen. Weber ging jedoch nie davon aus, die Bürokratie würde von sich aus reibungslos und ohne abzudriften funktionieren. Vielmehr zieht er selbst in Betracht, dass es zu informellen Mechanismen oder zu Improvisation kommen kann, was ja auch eher zu seiner Auffassung des sozialen Akteurs passt. Wie man den Untersuchungen Webers sinngemäß entnehmen kann, lässt sich eine Regel »managen«, 
und er erinnert zurecht daran, dass »die Amtsführung der Beamten [...] nach generellen, mehr oder minder festen und mehr oder minder erschöpfenden, erlernbaren Regeln « erfolgt. ${ }^{10}$ Obendrein kann die Einhaltung gesetzlicher Vorschriften ein Gegengewicht in Machtinteressen finden, auch sind die Akteure im Handlungsvollzug imstande, sich formal an eine Vorschrift zu halten, sie faktisch jedoch $\mathrm{zu}$ unterlaufen. ${ }^{11}$ Es ist durchaus wahrscheinlich, dass Weber, hätte er Le phénomène bureaucratique lesen können, in Crozier eher einen Fortsetzer denn einen radikalen Kritiker gesehen hätte. Ungeachtet all dessen sorgt die Bürokratie für Vorhersehbarkeit, auch wenn sie über Verabredungen funktioniert, insofern sich eine gewisse regelmäßige Wiederkehr von Verhaltensweisen einstellt. Wenn die Regel in den Augen der Handelnden als legitim erkannt wird, verpflichtet sie zum Handeln, gibt Verhaltensweisen vor. Die Frage lautet nicht, ob die Akteure die formalen Vorgaben passiv befolgen, so wie man es seiner Auffassung der rechtlichen Regel entnehmen könnte. Die Geltung einer Regel hängt davon ab, wie ein sozialer Akteur sie mehr oder weniger bewusst in sein eigenes situationsbezogenes Kalkül einbezieht. Genau deshalb kann Weber die Geltung der Regel sogar noch im Verhalten des Diebes ausmachen, der ja an ihr sein Verhalten orientieren muss. Gerade in der Ausrichtung des Verhaltens zeigt sich die Wirkkraft der Regeln. Doch über die stets singulären Modalitäten der Aneignung von Regeln durch die Individuen hinaus muss die kollektive Tragweite der Einhaltung von Regeln betont werden.

Formalismus und Abstraktheit von Regeln sind nicht nur nötig, um die sozialen Barrieren zu verschleiern, sie sind auch deren Regulationsprinzipien. Die Bürokratie stellt für Weber die Lösung für eine Welt dar, in der eine Vielfalt von Wertesystemen und sozialen Positionen herrscht. Sie ermöglicht die Durchsetzung von Disziplin in rationaler und einheitlicher Art und Weise. Unpersönlichkeit ist die Voraussetzung für Neutralität, insofern der Handelnde niemals durch seine soziale Basis determiniert wird. So verstanden, setzt Disziplin eine an Pflichtgefühl und vorbildlicher Rechtschaffenheit orientierte Ethik voraus, die aus dem Bürokraten einen - in Cromwells Worten - »Mann des Gewissens « und weniger der »Ehre« macht, dies in dem Sinne, dass Ehre stets einen gesellschaftlich festgelegten Wert darstellt. Weber erinnert daran, wenn er auf die Figur des Beamten zu sprechen kommt, dessen Gehorsam das Ergebnis der unpersönlichen Bindung an eine rechtlich definierte Amtspflicht ist; »[...] die unpersönliche Bindung an die generell bezeichnete sachliche >Amtspflicht< [...] ebenso wie das korrespondierende Herrschaftsrecht: - die `Kompetenz - [sind] durch rational gesatzte Normen (Gesetze, Verordnungen, Reglements) fest und [...] bestimmt $[\ldots] . \ll^{12}$ Recht und Bürokratie gehen überdies miteinander einher und funktionieren nach derselben Logik: Das formale Recht entspricht einer deduktiven Logik, in der das Besondere aus dem Allgemeinen abgeleitet wird, wobei die Bürokratie hinsichtlich der Qualifikation einer identischen Logik insofern entspricht, als die untergeordneten Qualifikationen aus den übergeordneten abgeleitet sind. Die bürokratische Hierarchie spiegelt nämlich die Abstufung der Kompetenzen wider und macht dadurch aus den Bürokratien transitive Hierarchien. Dieses Bemühen um die legitime Begründung der Bürokratie macht auch eine der Bedingungen ihrer Leistungsfähigkeit aus. Weber hat zudem seine Analyse der bürokratischen Verwaltung nicht von der der Herrschaft losgelöst. Daher stellt die Bürokratie in reiner Form die am besten für die Ausübung rational-legaler Herrschaft geeignete Verwaltungsweise dar.

7 In gewisser Weise kann man annehmen, dass die durch die Entwicklung der Bürokratie ausgelösten Ängste weitgehend dem im Grunde höchst revolutionären und innovativen 
Charakter dieses Organisationstypus geschuldet sind, über dessen Ausdehnung und tatsächliche Auswirkungen man aufgrund des durch ihn möglich gemachten vielfachen Machtzuwachses schwer Aussagen treffen konnte. Genau das hat auch die heftigen Befürchtungen Webers angesichts der unwiderstehlichen Ausweitung der Bürokratie genährt und ihn dazu geführt, die Ambivalenzen der Moderne zu betonen. Dieser Weitblick hing freilich mit der Zweigleisigkeit seines Untersuchungsansatzes zusammen, um die sich die Soziologen, insbesondere die Organisationssoziologen, im Allgemeinen herumgedrückt haben und die sich auf die im eigentlichen Sinne historische Dimension des Bürokratisierungsprozesses einerseits, auf die makrosoziale Dimension des bürokratischen Phänomens andererseits richtete.

Ich werde hier nicht nochmals auf den ersten Aspekt eingehen, auf dessen Bedeutung bei Weber ich bereits kurz hingewiesen habe. Indem man die Bürokratiestudien des deutschen Soziologen - was eigentlich noch schlimmer ist - vom Rest der von ihm entwickelten und viel umfassenderen Handlungssoziologie isolierte, hat man seine Soziologie der Bürokratie auf eine schlichte Spezialsoziologie reduziert, ohne dabei der tatsächlichen Reichweite seiner Interpretationen sowie des besonderen Stellenwerts der Bürokratie im Rahmen seiner Beweisführung gewahr zu werden. Die Soziologie hat die makrosoziale Unterfütterung der Weberschen Analyse aus den Augen verloren und die Herrschaft einzig unter dem organisationsinternen und funktionalen Aspekt, nämlich als die Bestimmung eines bloßen »Kontrollerfordernisses « analysiert, während die legal-rationale Herrschaftsweise sie doch an eine allgemeine legitime politische Ordnung knüpft, wie sie in der gesamten Gesellschaft zu erkennen ist, egal ob sie hier und dort mit Tradition oder Charisma gemischt ist oder nicht. Die Leistungsfähigkeit der Bürokratie liegt in ihrer Allgemeinheit und in dem Umstand, dass in dem Qualifikationsprinzip, das ihre zentrale Triebfeder darstellt, ein Klassifikations- wie ein Gerechtigkeitsprinzip zum Ausdruck kommen und miteinander verknüpft sind. Es steht daher insbesondere mit den praktischen Modalitäten der Herstellung von sozialen Hierarchien im Gleichklang, wie sie im Bildungssystem am Werke sind. Es rechtfertigt vollkommen die Hoffnung, die Weber wie Durkheim in es setzen konnten, ohne jemals die gesellschaftlichen Mechanismen zu verkennen, die sein Ideal pervertieren können.

9 Webers Insistieren auf die Herrschaftsmodelle hat manchen Beobachter verleitet, die idealtypische Darstellung einer besonderen Organisationsform mit der Realität eines Kollektivbetriebs zu verwechseln, ja gar den Idealtyp der Bürokratie mit einer normativen Vorgabe gleichzusetzen. In seiner mit Recht kritischen Bemerkung zielte Nisbet im Wesentlichen auf die Arbeiten amerikanischer Soziologen (unter denen Merton, Gouldner und Selznick die repräsentativsten sind), die das Webersche Bürokratiemodell als effiziente Verhaltenskontrolllogik untersuchten. Mit Ausnahme Selznicks interessierten sich Letztere nämlich kaum für die institutionelle Dimension der Bürokratie, die im Zentrum des Denkens des deutschen Soziologen steht. ${ }^{13}$ Die ohne ein rigides Klassensystem auskommende Bauweise der amerikanischen Gesellschaft wirkte sich derart aus, dass sie offenbar gar nicht auf die Idee kamen, die Bürokratie zuvorderst als das Mittel aufzufassen, sich um eines rationaleren Handelns willen sozialer Hierarchien zu entledigen. Die Organisationssoziologen lassen es im Übrigen meist bei der Frage der Rationalisierung formaler Organisationen bewenden, die unter dem Blickwinkel von Befehlsbeziehungen und der Hervorbringung effizienter Ordnungen wahrgenommen werden. Ihnen geht es um die Aufdeckung dessen, was ein stark formalisierter Typus von Arbeitsteilung an sozialen Beziehungsformen mit sich bringt und mit welchen Konsequenzen im Hinblick auf die Erledigung von Aufgaben 
dies erfolgt. Die an Weber geübten Kritiken beziehen sich diesbezüglich nur auf Teilaspekte, und die darin ausgedrückte Distanz ihm gegenüber ist in Wirklichkeit geringer, als ihnen bewusst ist. In dieser Hinsicht sind die in Frankreich von Michel Crozier angestoßenen Forschungen eine Fortführung der amerikanischen Forschungen. Selbst wenn er sich von der funktionalistisch ausgerichteten Analyse von Organisationen distanziert und einen sich auf die Strategien der Akteure innerhalb von organisierten Ganzheiten stützenden Ansatz vorschlägt, macht Crozier hier keineswegs eine Ausnahme: Auch sein Interesse ist durch die alleinige Berücksichtigung von Teilaspekten der Weberschen Bürokratietheorie ganz klar eingeschränkt. Bürokratie ist für ihn nur eine Formalisierung sozialen Austauschs mittels Systemen von Regeln und Verfahrensweisen, an die sich die Akteure halten müssen. Nicht zufällig weisen die meisten Untersuchungen eine Gemeinsamkeit auf, nämlich dass sie auf eine Kritik der Bürokratie hinauslaufen, wie sie Weber modellhaft dargestellt hat, indem er auf die »Teufelskreise« hinwies, die ihrer Leistungsfähigkeit zuwiderlaufen. Gewiss müsste man die Analyse je nach Autor verfeinern, doch lässt sich trotz allem sagen, dass die Bürokratiesoziologie ganz allgemein gesprochen bei einer Soziologie »organisierter Arbeit« stehen geblieben ist. In der Tat hat sich die Organisationssoziologie weniger vom Weberschen Vorbild freigemacht als dieses vielmehr fortgeführt, indem sie das Funktionieren von Bürokratien in der Praxis aufdeckte, mit dem sich Weber um so weniger aufgehalten hat, als es nicht im Zentrum seiner Interessen stand.

Die Verwechslung der Bürokratie mit ihrem Idealtypus ist nicht folgenlos geblieben. Ihretwegen wurden bisweilen nicht nur viele Dummheiten geschrieben, sie hat darüber hinaus auch die besonderen Stärken des Weberschen Programms verdeckt, nämlich den Zusammenhang, den Weber zwischen der Bürokratie und einer Form legitimer Herrschaft herstellt, die über sie hinausgeht und ihr zugleich Vorschub leistet. Die Kritik hat dazu geführt, dass man die Bürokratie nur an der Elle der Rigidität ihres Funktionierens maß, einer Rigidität, die sie nur um den Preis der Ausbildung einer Informalität ablegen kann, die dann als vorteilhaft gilt, wenn sie für Handlungsflexibilität sorgt.

11 Formale Organisationen werden meist als Komplexe von Aufgaben definiert, die intentional gegliedert sind, um unter Annahme besonderer Hypothesen bezüglich der relevanten Wirkungsverhältnisse vorgegebene Ziele zu erreichen. Das Informelle wird oft als unabdingbar für die Anpassungsleistungen von Organisationen angesehen, selbst wenn man den »Informalitäten« ursprünglich nur deshalb Aufmerksamkeit gewidmet hat, weil sie in formalen Organisationen für Probleme sorgen. So laufen für Roethlisberger und Dickson informelle Beziehungen darauf hinaus, Individuen und Untergruppen vor dem Zugriff der formalen Organisation zu schützen..$^{14}$ Merton, Selznick und Gouldner sehen im Allgemeinen in informellen Beziehungen den Ausdruck latenter Funktionen, die mit dem Unvermögen formaler Strukturen, die Bedürfnisse der Individuen zu berücksichtigen, zusammenhängen. Für Barnard sorgen informelle Organisationen für eine effektive Kommunikation in der Organisation, die die Fähigkeit der Organisation verbessert, sich zu einem effektiven Kooperations- oder Koordinationssystem zu entwickeln, wobei mit Kooperation zugleich ein Prozess und ein Ziel gemeint ist. ${ }^{15}$ Kurzum, man sieht, dass die Entwicklung informeller Mechanismen häufig mit dem Scheitern und den Unzulänglichkeiten der formalen Organisation in Zusammenhang gebracht wird. Das begründete den besonderen Blick des Soziologen darauf. Er entschlüsselte eine Realität, machte sie sicht- und beobachtbar. Er sorgte für »Enthüllungen«, und man brauchte ihn, um die eigentlichen 
Spielregeln kennenzulernen. Im Grunde war er so etwas wie ein Organisationsethnograph. Die Bürokratie wollte nämlich für die Vorhersehbarkeit von sozialem Verhalten mittels eines Satzes von so präzise wie möglich definierten Regeln sorgen. Diese wurden im Allgemeinen nicht wörtlich befolgt, was zwar eine gewisse Intransparenz, jedoch nicht unbedingt Unvorhersehbarkeit nach sich zog. Tatsächlich wird das Informelle weitgehend durch das Formelle strukturiert, mangelnde Vorhersehbarkeit gibt es nur für diejenigen, die die impliziten Regeln des Spiels nicht durchschauen.

Ohne dass man den Ursprung des sich in jeder Organisation stellenden Problems verstanden hat, nämlich wie mit der mehr oder weniger ausgeprägten Spannung zwischen Ordnung und Handeln umgegangen werden soll, wird oft ein Gegensatz zwischen der »Rigidität von Strukturen« und der empirisch beobachtbaren »Flexibilität im Handeln« aufgebaut. Viele, vor allem verwaltungswissenschaftliche Untersuchungen gefallen sich förmlich darin, einander widersprechende Visionen von Organisationen zu entwerfen. ${ }^{16}$ Aus diesem Blickwinkel betrachtet, stellt der Umgang mit dem sozialen Status der Mitglieder einer Organisation und der Legitimität der Hierarchieebenen ein ganz anderes Problem dar als die Erledigung von präzisen, auf die Lösung von praktischen Fragen zielenden Aufgaben. Das Ineinandergreifen der beiden Ebenen kann man niemals völlig unter den Tisch fallen lassen: Einerseits ist eine Organisation eine Form des Umgangs mit sozialen Beziehungen und damit der Rechtfertigung der ungleichen Verteilung von Positionen, andererseits stellt sie ein Universum von Aufgaben dar, die die Akteure erledigen müssen und deren Verknüpfung die Produktion bestimmt. Die zentrale Frage ist letztlich die nach dem Umgang mit dieser Dualität. Das Leistungsvermögen der Bürokratie bestand historisch gesehen in der völligen Übereinstimmung von Ordnungs- und Handlungsprinzipien, von Autorität und Macht. Das Funktionieren der Organisationen hängt mehr und mehr von der Fähigkeit ab, diese beiden Dimensionen gleichzeitig und so, dass sie miteinander vereinbar sind, zu behandeln.

Wie steht es nämlich darum, wenn Ordnung und Disziplin erzeugt und zugleich Lösungen für praktische Probleme gefunden werden müssen, die die Hierarchieebenen und organisatorischen Trennlinien zunehmend überschreiten? Das gilt ebenso für die Unternehmenswelt, wo der Begriff des erweiterten Unternehmens neue Formen der Kooperation erfordert, wie für die öffentlichen Bürokratien, wo die Beschaffenheit der öffentlichen Probleme sowie die Definition öffentlichen Handelns zunehmend weniger mit der institutionellen Architektur des Staates übereinstimmen. Die herausragende Stellung der Handlungsprobleme - das Privileg des Handelns überhaupt - hat in einem solchen Maße dazu geführt, einen Gegensatz zwischen der managerialen Tugend der transversalen Kooperation und der Schwerfälligkeit der prozeduralen Koordination aufzubauen, dass - wenn man die Organisation allein in der Bürokratie aufgehen ließ die Versuchung groß war, Handeln und Organisation einander gegenüberzustellen, was implizit einen Bedeutungsverlust der organisationellen Frage nach sich zog.

In Frankreich hat sich die weitgehend an Crozier orientierte Organisationssoziologie umso leichter dazu entschlossen, als die von Crozier vorgeschlagene und entwickelte Herangehensweise sich mehr auf den Akteur als auf die Organisation stützt. Es ist absolut symptomatisch, dass sich die drei Fragen, die Crozier zufolge die Grundlage seiner Überlegung bildeten, kaum spezifisch auf die Organisation richten, nämlich wie menschliche Wesen dazu kommen, das Problem ihrer Kooperation zu lösen, welche 
Fähigkeiten die verschiedenen Lösungen jeweils erfordern und was der Preis dafür ist. Für ihn ist die Organisation höchstens ein Handlungskontext, in dem Sinne, als sie die Ressourcen und Zwänge an die Akteure heranträgt, deren Spiel weniger durch die Organisationen an sich bestimmt wird, als durch die Herausforderungen, denen sie sich stellen müssen. ${ }^{17}$ Die Croziersche Soziologie ist weniger eine Organisationssoziologie als eine Soziologie des "Handelns im organisierten Kontext«. Nicht zufällig weiteten sich die vom Centre de sociologie des organisations vorgelegten Studien auf umfassendere Entitäten als nur Organisationen aus, ob es sich um das politisch-administrative System Frankreichs oder die Funktionsweise von Märkten handelt. Das Konzept des " konkreten Handlungssystems", wie es in L'acteur et le système ausgearbeitet wird, verbannt faktisch dasjenige der Organisation. ${ }^{18}$ Die Realität der Handlungssysteme wirkt sich unter dem Handlungsaspekt letztlich stärker determinierend aus als die "Strukturen" der Organisationen. Der auf die Handlung gelegte Akzent erlaubt es gewiss, sich dem Zugriff der Organisation zu »entziehen« und sich eines komplexeren Universums wechselseitiger Abhängigkeit, wie es das konkrete Aufzeigen von Handlungssystemen offenbart, bewusst zu werden. Auch wenn die Analyse eine detailliertere Ausarbeitung verdiente, kann man gleichwohl hervorheben, dass man dadurch ungewollt den Anschein erwecken könnte, die Kontingenz und Zufälligkeit der Welt erklärten sich allein durch den fortwährend "ausgehandelten « Charakter der sozialen Ordnung. Tatsächlich lässt sich die allmähliche Auflösung der Organisation auf eine überzogen prozessuale Auffassung der Sozialwelt in Verbindung mit einer kritikwürdigen Konzeption von Macht zurückführen, die die strukturelle Dimension sozialen Handelns aus dem Blick verliert. ${ }^{19}$ Weil sie allzu sehr auf die Handlungssysteme Nachdruck legt, ist die Organisationssoziologie heutzutage zu einer übertriebenen Aufweichung des Phänomens Organisation gelangt. Die Radikalisierung der Analyse hat sogar zu einer völligen Auflösung geführt, die die einleuchtendsten Lehren Webers aus dem Blick verloren hat.

Dennoch führt die Analyse sozialer Prozesse letztendlich dazu, sich die Frage nach dem Status der Organisation zu stellen. Das Aufzeigen von Handlungssystemen kann nicht vergessen machen, dass die Organisation zugleich als Entwurf und als für die Handlung notwendige Vermittlung existiert. Das Risiko eines im Wesentlichen "strategieorientierten" Ansatzes besteht ganz klar darin, dass die Bedeutung der organisatorischen Formalisierung minimiert wird und dass man daraus nur noch einen mehr oder weniger einengenden Rahmen macht, innerhalb dessen sich das Wechselspiel der Akteure entwickelt. Wie Éraly bemerkt, ist die Struktur nur mehr ein im Dienste der Interessen der Akteure stehendes, zufällig zustande kommendes Gefüge. Die Organisation hat damit jegliche institutionelle Dimension und jede Verbindung zur Geschichte verloren. ${ }^{20}$ Die These, eine Organisation sei ein "soziales und kulturelles Konstrukt«, zeigt dennoch deutlich, dass eine Organisation stets einem bewusst und freiwillig unternommenen Bemühen um die Strukturierung des kollektiven Handelns entspricht, dass sie an ein Projekt gebunden ist, das ihr Sinn verleiht. Der bekundete Wille, eine "Dekonstruktion" der Organisation vorzunehmen, kann kein Hindernis dafür darstellen, dass man sich mit gutem Recht fragt, warum die sozialen Akteure einen gut Teil ihrer Zeit damit zubringen, Organisationen zu »konstruieren«. Hier droht eine Verwechslung zweier klar voneinander getrennter Realitätsebenen, der Ordnung und des Handelns, deren Verknüpfung sich stets als problematisch erweist. Einerseits mobilisiert die Anfertigung (confection) einer Organisation (so möchten wir die Vorstellung eines design übersetzen, um deutlich zu machen, dass es um den 
"Entwurf« [dessin] der Organisation und gleichzeitig um die Aktivität des »Entwerfens« [dessiner] geht) den Willen der Akteure, Wissensformen, Handlungsgrundsätze und soziale Verhältnisse, die zum Entwurf (projection) einer besonderen Lösung führen. Auf der anderen Seite steht die Analyse der Funktionsweise eines Netzwerks von Akteuren, die von einem bestimmten Problem betroffen sind. Sie läuft darauf hinaus, ein System von Akteuren offenzulegen, deren Grad an wechselseitiger Abhängigkeit nicht in direktem Zusammenhang mit den institutionalisierten Grenzen steht. Dass eine solche Analyse möglicherweise dazu führt, die Angemessenheit eines bestimmten organisatorischen Zuschnitts infrage zu stellen, kommt nicht notwendigerweise einer Verdammung der Organisation als solcher gleich. Sie kann umgekehrt eine Umgestaltung der Arbeitsorganisation nach sich ziehen, auch wenn es ebenso zutrifft, dass sich zu einem gegebenen Zeitpunkt die Frage der Legitimität und der Eigenart einer Institution in Bezug auf ihre organisatorische Leistungsfähigkeit stellt. Wie ersichtlich, kann die Analyse zu einem Mittel werden, die Organisation neu zu denken. Weder Institution noch Organisation lassen sich in ein Handlungssystem auflösen. Man muss wohl zugestehen, dass zwischen Organisation und Institution oft nur eine ganz schmale Grenze verläuft. Es ist aber nicht so, dass man, weil sich Akteursnetzwerke mit über die Organisationsgrenzen hinausreichenden Beziehungsnetzen ausmachen lassen, jegliche Formalisierung verwerfen sollte, die man etwas vorschnell mit dem bürokratischen Modell gleichzusetzen versucht ist. Man kann die Bürokratie nicht einfach aufgrund des Vorliegens funktionaler Beziehungen ablehnen. Überdies werden vielfach in übertriebener Weise Organisation und Bürokratie in einen Topf geworfen. Nun ähnelt aber die Demontage des klassischen Organisationsbegriffs, wie Friedberg sie betreibt, in großem Maße einer Kritik am bürokratischen Modell. ${ }^{21}$ Wie auch immer es um die Gültigkeit und die Legitimität eines solchen Vorgehens bestellt sein mag, es ist eine Quelle von Konfusion. Dass man Einwände gegenüber dem Festnageln der Organisation auf eine im Wesentlichen bürokratische Kennzeichnung hat, erklärt keineswegs, warum man diese Ablehnung auf jede Form von Organisation ausdehnen sollte. Die Frage ist vielmehr, ab welchem Moment die Bürokratie nicht mehr die adäquate Form von Arbeitsteilung, aber auch von Autorität darstellt. Wenn die Bürokratie tot ist, welches sind dann die Formen, die das organisierte Handeln oder genauer das freiwillige Handeln in puncto kollektiver Kooperation annehmen muss, auf welche Art und Weise können menschliche Aktivitäten reguliert werden, schließt man einmal den Rückgriff auf Gefühle und den Patrimonialismus aus? Wenn die Bürokratie fortbesteht, dann deshalb, weil sich mit ihr soziale Ordnung begründen und damit soziale Hierarchien nach dem schwer angreifbaren bzw. vollkommen ersetzbaren Grundsatz der Kompetenz handhaben lassen. Die Bürokratie ist, wie Weber gesehen hat, Ausdruck einer legitimen Ordnung sozialer Beziehungen, das macht ihre Überwindung stets zu einer heiklen Angelegenheit, weil man dann alternative Kriterien angeben müsste. Die Schwierigkeit, solche zu nennen, erklärt weitgehend, dass die Gegenüberstellung mit dem Weberschen Idealtypus der Bürokratie unumgänglich bleibt. $^{22}$

Heute vollzieht sich die Kombination von Ordnung und Handeln über die Verknüpfung dessen, was die Angelsachsen »tightly coupled systems« nennen (also Bürokratien, deren Funktion darin besteht, über die Ressourcen für Handlungen zu verfügen), mit "loosely coupled systems«, lose verbundenen Handlungssystemen zur Problembehandlung ohne echte Zentralautorität, deren Interaktion und Kommunikation durch die Eigenart der zu behandelnden Probleme diktiert werden und 
nicht durch eventuelle a priori erstellte Organigramme. ${ }^{23}$ Wenn sich die bürokratische Logik für den Umgang mit Handlungsproblemen nicht völlig eignet, wenn man aber trotzdem die Fähigkeit zur Lösung von Problemen (problem-solving capacity) und die Fähigkeit zum Umgang mit Menschen (control capacity) weiterhin zusammendenken will, ist das soziale Funktionieren mehr und mehr vom Grad der Vereinbarkeit bei der Bearbeitung dieser beiden Probleme abhängig: Gefragt ist also die Erfindung einer »Regulierung im Verbund «, um eine äußerst hilfreiche Formulierung von Jean-Daniel Reynaud aufzugreifen. ${ }^{24}$ Die Zukunft der Bürokratie liegt nicht im Fortbestand ihrer Formen, sie liegt in der Möglichkeit oder Unmöglichkeit, Legitimität und Leistungsfähigkeit miteinander $\mathrm{zu}$ verbinden, also eine legitime Ordnung für das Handeln hervorzubringen.

Das Anfertigen von Organisationen bleibt im gleichen Zug ein Handlungsproblem. Nicht zufällig stellt sich die Frage der Bürokratie erneut zu einer Zeit tiefer Unsicherheit hinsichtlich der Architektur der Staaten und ihrer Verwaltungen, aber auch der Unternehmen. Die Formalisierung oder zumindest die Offizialisierung der Verfahren bedeutet freilich nicht, dass man stets ausschließlich die Prinzipien der Bürokratie gutheißen müsste, ebenso wenig dass es ausgemacht ist, dass wir auf jeden Fall mit der Bürokratie brechen müssten. Die einzige Gewissheit, die wir haben, ist, dass wir die Organisation nicht ignorieren können.

\section{BIBLIOGRAPHIE}

\section{Werke Max Webers}

GARS: Weber, M. (1988): Gesammelte Aufsätze zur Religionssoziologie, Bd. 1, 9. Aufl., Tübingen: J. C. B. Mohr.

GPS: Weber, M. (1988): Gesammelte Politische Schriften, hrsg. v. J. Winckelmann, 5. Aufl., Tübingen: J. C. B. Mohr.

MWG I/15: Weber, M. (1984): Max Weber-Gesamtausgabe, Bd. 15, Abteilung I: Zur Politik im Weltkrieg. Schriften und Reden 1914-1918, hrsg. v. W. J. Mommsen, in Zusammenarbeit mit G. Hübinger, Tübingen, J. C. B. Mohr.

MWS I/19: Weber, M. (1991): Die Wirtschaftsethik der Weltreligionen I. Konfuzianismus und Taoismus, Max Weber-Studienausgabe Bd. I/19, hrsg. v. H. Schmidt-Glintzer, in Zusammenarbeit mit P. Kolonko, Tübingen: J. C. B. Mohr.

WL: Weber, M. (1988): Gesammelte Aufsätze zur Wissenschaftslehre, hrsg. v. J. Winckelmann, 7. Aufl., Tübingen: J. C. B. Mohr.

WuG: Weber, M. (1972): Wirtschaft und Gesellschaft, hrsg. von J. Winckelmann, 5. rev. Aufl., Tübingen: J. C. B. Mohr. 


\section{Sekundärliteratur}

Barnard, C. (1971): The Functions of the Executives, Cambridge, Mass.: Harvard University Press.

Bézès, P. (2007): „Construire des bureaucraties wébériennes à l'ère du New Public Management?«, Critiques internationales, Bd. 2, 35, S. 9-29.

Chazel, F. (1994): »L'action organisée et l'analyse sociologique contemporaine«, Sociologie du travail, 1: »Symposium sur Le Pouvoir et la Règle».

Chazel, F. (2000 [1995]): »Éléments pour une reconsidération de la conception wébérienne de la bureaucratie«, in: ders. (2000): Aux fondements de la sociologie, Paris: PUF; in dieser Nummer von Trivium unter dem Titel »Webers Bürokratiebegriff - neu betrachtet«.

Crozier, M. (1970-1971): »Sentiments, organisations et systèmes«, Revue française de Sociologie, XIXII, Sondernummer.

Crozier, M. / Friedberg, E. (1993 [1977]): Die Zwänge kollektiven Handelns. Über Macht und Organisation. Übers. von E. Friedberg u. S. Stelzer, Neuausgabe, Frankfurt am Main: Hain.

De Coster, M. (1978): L'analogie en sciences humaines, Paris: PUF.

Dubet, F. (1984): Sociologie de l'expérience, Paris: Seuil.

Dupuy, F. / Thœnig, J.-C. (1983): Sociologie de l'administration française, Paris: Armand Colin.

Duran, P. (2009): »Légitimité, droit et action publique«, L'année sociologique, Sondernummer »Pour une sociologie politique du droit«, hrsg. von P. Duran und J. Commaille, Bd. 59, 2.

Éraly, A. (1988): La structuration de l'entreprise, La rationalité en action, Brüssel: Editions de l'Université libre de Bruxelles.

Friedberg, E. (1995 [1993]): Ordnung und Macht. Dynamiken organisierten Handelns. Aus dem Französischen von G. Voß, Frankfurt am Main u. a.: Campus.

Giddens, A. (1979 [1973]): Die Klassenstruktur fortgeschrittener Gesellschaften, übers. von C. Stephan, Frankfurt am Main: Suhrkamp.

Giddens, A. (1981): A Contemporary Critique of Historical Materialism, Bd. I: Power, Property and the State, London: Macmillan.

Le Galès, P. / Scott, A. (2008): „Une révolution bureaucratique? Autonomie sans contrôle ou >free markets, more rules`«, Revue française de sociologie, Bd. 49, 2, S. 301-330.

March, J. G. / Simon, H. A. (Hg.) (1979 [1958]): Les organisations, Paris: Dunod; engl. Original: Organizations, New York: Wiley; 2. Aufl., Cambridge, Mass. [u. a.]: Blackwell 1994.

Nisbet, R. A. (1984 [1966]): La tradition sociologique, Paris: PUF; engl. Original: The Sociological tradition, New York: Basic books.

Reynaud, J.-D. (1988): »La régulation dans les organisations: régulation de contrôle et régulation autonome«, Revue française de sociologie, Bd. XXIX.

Roethlisberger, F. J. / Dickson, W. J. (1947): Management and the Worker, Cambridge, Mass.: Harvard University Press.

Selznick, P. (1949): TVA and the Grass Roots, New York: Harper \& Row. 


\section{NOTES}

1. Nisbet (1984 [1966]), S. 180.

2. Chazel (2000 [1995]); siehe auch diese Nummer von Trivium.

3. March / Simon (1979 [1958]).

4. Chazel (2000 [1995]), S. 180.

5. Chazel (2000 [1995]).

6. Giddens (1979 [1973]) und Giddens (1981).

7. WuG. Man kann aber auch heranziehen »Wissenschaft als Beruf« (WL) und GPS.

8. Zum Beispiel De Coster (1978).

9. Nisbet (1984 [1966]).

10. Vgl. »Wesen, Voraussetzungen und Entfaltung der bürokratischen Herrschaft« (WuG, S. 551579), hier S. 552, sowie »Parlament und Regierung im neugeordneten Deutschland« (GPS, S. 306443; auch MWG I/15, S. 432-596).

11. WuG, S. 197.

12. GARS, S. 272; auch MWS I/19, S. 24).

13. Es sei dennoch hinzugefügt, dass Selznick sich für institutionelle Normen nur als Handlungszwänge interessiert. Vgl. Selznick (1949). Selznick ist wahrscheinlich der Autor, auf den diese Kritiken am wenigsten zutreffen.

14. Roethlisberger / Dickson (1947).

15. Barnard (1971).

16. Sehr bezeichnend in dieser Hinsicht Dupuy / Thœnig (1983).

17. Crozier (1970-1971).

18. Crozier / Friedberg (1993).

19. Vgl. zu diesem Punkt die heftige Kritik von Chazel (1994) sowie Dubet (1984).

20. Éraly (1988).

21. Friedberg (1995 [1993]).

22. Vgl. beispielsweise die erhellenden Artikel von Bézès (2007) sowie von Le Galès / Scott (2008). 23. Duran (2009).

24. Ich mache hier eine Anleihe bei Jean-Daniel Reynaud, der das Vorliegen einer zweifachen Regulation in den Organisationen klar erkannt hat, und versuche, seine Gedanken weiterzuführen. Vgl. Reynaud (1988).

INDEX

Schlüsselwörter : Webers Bürokratietheorie, allgemeine Handlungstheorie, Organisationssoziologie, Herrschaft, Macht

Mots-clés : théorie weberienne de la bureaucratie, théorie générale de l'action, sociologie des organisations, autorité, pouvoir 


\section{AUTEURS}

\section{PATRICE DURAN}

Patrice Duran ist Professor an der Ecole normale supérieure in Cachan. Nähere Informationen

finden Sie hier. 